\title{
SOME RESULTS CONCERNING THE BEHAVIOR AT INFINITY OF REAL CONTINUOUS SOLUTIONS OF ALGEBRAIC DIFFERENCE EQUATIONS ${ }^{1}$
}

\author{
OTIS E. LANCASTER ${ }^{2}$
}

The behavior at infinity of real continuous solutions of algebraic differential equations has been studied by Borel, Lindelöf, Hardy, Fowler, and Vijayaraghavan,,$^{3}$ but, as far as the author is aware, the corresponding problem for difference equations has not been considered, except for the special case of solutions in the neighborhood of a double point. ${ }^{4}$

In this paper we propose to study the rate of increase, as the independent real variable $x$ becomes infinite, of real continuous solutions of algebraic difference equations: that is, of equations of the form

$$
P(y(x+m), y(x+m-1), \cdots, y(x), x)=0,
$$

where $P$ is a polynomial with real coefficients in its arguments $y(x+m), y(x+m-1), \cdots, y(x)$, and $x$. Among the terms of the polynomial $P$, there is a term

$$
T^{\prime}=A^{\prime} x^{\alpha^{\prime}} y(x)^{\beta_{0}{ }^{\prime}} y(x+1)^{\beta_{1}^{\prime}} \cdots y(x+m)^{\beta_{m^{\prime}}},
$$

which has the property that if

$$
T=A x^{\alpha} y(x)^{\beta_{0}} y(x+1)^{\beta_{1}} \cdots y(x+m)^{\beta_{m}}
$$

1 Presented to the Society, December 30, 1937.

${ }^{2}$ I am indebted to Professor G. D. Birkhoff for counsel and encouragement during the preparation of this paper.

${ }^{3}$ E. Borel, Mémoire sur les séries divergentes, Annales de l'École Normale Supérieure, Paris, (3), vol. 16 (1899), p. 26 ff.; E. Lindelöf, Sur la croissance des intégrales des équations différentielles algébriques du premier ordre, Bulletin de la Société Mathématique de France, vol. 27 (1899), pp. 205-215; G. H. Hardy, Some results concerning the behavior at infinity of a real and continuous solution of algebraic differential equations of the first order, Proceedings of the London Mathematical Society, (2), vol. 10 (1912), pp. 451-468; R. H. Fowler, Some results on the form near infinity of real continuous solutions of a certain type of second order differential equations, Proceedings of the London Mathematical Society, (2), vol. 13 (1914), pp. 341-371; T. Vijayaraghavan, Sur la croissance des fonctions définies par les équations différentielles, Comptes Rendus de l'Académie des Sciences, Paris, vol. 194 (1932), pp. 827-829.

${ }^{4}$ If $\lim _{x \rightarrow \infty} u(x)=U$, then $U$ is a double point of the difference equation $u(x+p)=f[u(x+p-1), u(x+p-2), \cdots, u(x)]$, when it is a root of the algebraic equation $U=f(U, U, \cdots, U)$. See S. Lattès, Sur les suites récurrentes non linéaires et sur les fonctions génératrices de ces suites, Annales de la Faculté des Sciences de Toulouse, (3), vol. 3 (1911), pp. 75-124; J. Horn, Zur Theorie der nicht linearen Differential- und Differenzengleichungen, Journal für die reine und angewandte Mathematik, vol. 141 (1912), pp. 182-216. 
is any other term of the polynomial $P$, then in the sequence of differences

$$
\beta_{m}^{\prime}-\beta_{m}, \beta_{m-1}^{\prime}-\beta_{m-1}, \cdots, \beta_{1}^{\prime}-\beta_{1}, \beta_{0}^{\prime}-\beta_{0}, \alpha^{\prime}-\alpha
$$

the first nonzero term is positive. We shall call $T^{\prime}$ the principal term of the equation. The proofs of the first four theorems are based upon the limits, as $x \rightarrow \infty$, of the ratios of the principal term to the other terms of the difference equation.

For the convenience of expressing the iterates of the logarithm and the exponential we shall use the notation that was employed by Hardy in his book Orders of Infinity, namely,

$$
\begin{aligned}
e_{1}(v) & =\exp (v), & e_{n}(v) & =\exp \left[e_{n-1}(v)\right], \\
l_{1}(v) & =\log (v), & l_{n}(v) & =\log \left[l_{n-1}(v)\right] .
\end{aligned}
$$

THEOREM 1. A real continuous solution of an algebraic difference equation of the first order cannot equal or exceed the function $\mathrm{Ce}_{2}\left[x l_{n}(x)\right]$ for all $x>x_{0}(n)$, where $C$ is any positive constant and $n$ is any integer.

Proof. First, assume that $\mathrm{Ce}_{2}\left[x l_{n}(x)\right]$ is a solution of the first order difference equation (1). Divide the equation by $T^{\prime}$ and replace $y(x)$ by $\mathrm{Ce}_{2}\left[x l_{n}(x)\right]$. Then, all terms except one, $T^{\prime} / T^{\prime}$, are of the form $T / T^{\prime}$, where

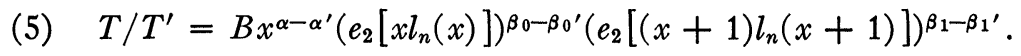

These terms $T / T^{\prime}$ are of three possible types:

$$
\begin{array}{lr}
B\left\{\frac{x^{K_{0}}\left(e_{2}\left[x l_{n}(x)\right]\right)^{K_{1}}}{e_{2}\left[(x+1) l_{n}(x+1)\right]}\right\}^{\beta_{1}^{\prime}-\beta_{1}}, & \beta_{1}^{\prime}>\beta_{1} ; \\
B\left\{\frac{x^{K_{2}}}{e_{2}\left[x l_{n}(x)\right]}\right\}^{\beta_{0}^{\prime}-\beta_{0}}, & \beta_{1}^{\prime}=\beta_{1}, \beta_{0}^{\prime}>\beta_{0} ; \\
B x^{\alpha-\alpha^{\prime}}, & \beta_{1}^{\prime}=\beta_{1}, \beta_{0}^{\prime}=\beta_{0}, \alpha^{\prime}>\alpha,
\end{array}
$$

where $K_{0}, K_{1}, K_{2}$ are rational numbers. The limit of each of these expressions as $x \rightarrow \infty$ is zero. Hence, we have a contradiction to the assumption that $y=\mathrm{Ce}_{2}\left[x l_{n}(x)\right]$ is a solution of (1). For there exists an $x_{0}(n)$ such that for $x>x_{0}(n)$ the sum of all the terms $T / T^{\prime}$ is less than one in absolute value, whereas $T^{\prime} / T^{\prime}=1$.

Second, a function $y(x)$ which is greater than $C e_{2}\left[x l_{n}(x)\right]$ for $x>x_{0}(n)$ cannot $^{5}$ be a solution of the first order difference equation

5 The symbol $x_{0}(n)$ does not always denote the same value. In fact, it may represent several different values within one proof. 
(1). For assume that $y(x)$ is such a solution of (1); then the ratios $T / T^{\prime}$ are again of the three types:

$$
\begin{array}{ll}
B\left\{\frac{x^{K_{0}} y(x)^{K_{1}}}{y(x+1)}\right\}^{\beta_{1^{\prime}-\beta_{1}},}, & \beta_{1}^{\prime}>\beta_{1}, \\
B\left\{\frac{x^{K_{2}}}{y(x)}\right\}^{\beta_{0}^{\prime}-\beta_{0}}, & \beta_{0}^{\prime}>\beta_{0}, \\
B x^{\alpha-\alpha^{\prime}}, & \alpha^{\prime}>\alpha .
\end{array}
$$

The ratios of types $\left(b^{\prime}\right)$ and $\left(c^{\prime}\right)$ approach zero, as $x$ becomes infinite, for all rational values of $K_{2}$; hence, if $y(x)$ is a solution, not all of the ratios of type $\left(\mathrm{a}^{\prime}\right)$ may approach zero, as $x \rightarrow \infty$, or we have a contradiction as above. Thus there is at least one ratio of type $\left(a^{\prime}\right)$ for which $K_{1}>0$. There may be several such ratios; however, the one which has the maximum value of $K_{1}$ is for all $x>x_{0}(n)$ greater in absolute value than any of the others. Hence if $M_{1}$ denotes this maximum value of $K_{1}$, then

$$
\frac{x^{K_{0}} y(x)^{M_{1}}}{y(x+1)}>\frac{1}{N}
$$

for all $x$ greater than or equal to $x_{0}(n)$. From this there follow in succession

$$
\begin{array}{lr}
y(x+1) \leqq N x^{K_{0}} y(x)^{M_{1}} & \begin{array}{r}
x \geqq x_{0}, \\
y\left(x_{0}+1\right) \leqq N\left[x_{0} y\left(x_{0}\right)\right]^{M},
\end{array} \\
\cdot \cdot \cdot \cdot \cdot \cdot \cdot \cdot \cdot \cdot \cdot \cdot \cdot \cdot \cdot, & \text { where } M \geqq K_{0}, M_{1}, 2, \\
y\left(x_{0}+p\right) \leqq\left[N y\left(x_{0}\right)\right]^{M^{p}}\left(x_{0}+p\right)^{M^{p+1},} & p=1,2,3, \cdots \cdot
\end{array}
$$

Therefore,

(8) $\lim _{p \rightarrow \infty} \frac{y\left(x_{0}+p\right)}{e_{2}\left[\left(x_{0}+p\right) l_{n}\left(x_{0}+p\right)\right]} \leqq \lim _{p \rightarrow \infty} \frac{\left[N y\left(x_{0}\right)\right]^{M^{p}}\left(x_{0}+p\right)^{M^{p+1}}}{e_{2}\left[\left(x_{0}+p\right) l_{n}\left(x_{0}+p\right)\right]}=0$.

This contradicts the assumption that $y(x)$ is greater than $C e_{2}\left[x l_{n}(x)\right]$. Thus, no continuous function $y(x), y(x) \geqq C e_{2}\left[x l_{n}(x)\right]$ for all $x>x_{0}(n)$, may be a solution of (1).

It is important to note that the above theorem does not state that a solution of a first order difference equation may not exceed $C e_{2}\left[x l_{n}(x)\right]$ at an infinite number of points $x_{1}, x_{2}, x_{3}, \cdots, x_{i} \rightarrow \infty$. However, it does follow from the proof that if a solution $y(x)$ equals $e_{2}\left[x l_{n}(x)\right]$ at a point $x>x_{0}(n)$, then $y(x+1)<e_{2}\left[(x+1) l_{n}(x+1)\right]$. 
Thus a solution $y(x)$ cannot exceed $e_{2}\left[x l_{n}(x)\right]$ over an interval of difference, ${ }^{6}$ for $x>x_{0}(n)$.

Although we have not been able to generalize Theorem 1 so as to include all algebraic difference equations of the $m$ th order, we have been able to establish the following facts:

THeORem 2. The function $\mathrm{Ce}_{2}\left[x l_{n}(x)\right],(C \neq 0)$, cannot be a solution of an algebraic difference equation for any value of $n$.

The proof is similar to the first part of Theorem 1. Under the assumption that the theorem is false, $y(x)=C e_{2}\left[x l_{n}(x)\right]$ is a solution of (1) and

$$
\frac{T}{T^{\prime}}=B x^{\alpha-\alpha^{\prime}} \prod_{j=0}^{m}\left\{e_{2}\left[(x+j) l_{n}(x+j)\right]\right\}^{\beta_{j}-\beta_{j^{\prime}}} .
$$

The considerations of the limits of these ratios divide into $m+2$ cases corresponding to the $m+2$ possible relations (4) between the exponents of the principal term and those of the other terms of (1). In the $(m+1-i)$ th case, $\beta_{m}{ }^{\prime}=\beta_{m}, \beta_{m-1}{ }^{\prime}=\beta_{m-1}, \cdots, \beta_{i+1}{ }^{\prime}=\beta_{i+1}, \beta_{i}^{\prime}>\beta_{i}$, the ratios are of the form

$$
B\left\{\frac{x^{K} \prod_{j=0}^{i-1}\left\{e_{2}\left[(x+j) l_{n}(x+j)\right]\right\}^{K_{j+1}}}{e_{2}\left[(x+i) l_{n}(x+i)\right]}\right\}^{\beta_{i^{\prime}-\beta_{i}}},
$$

where the $K_{v},(v=0,1,2, \cdots, i)$, are rational numbers. The limit, as $x$ becomes infinite, of such a quotient is zero for $i=0,1,2, \cdots, m$ regardless of the magnitudes of the $K_{v}$. In the $(m+2)$ nd case, the ratios also approach zero for they are of the form $B x^{\alpha-\alpha^{\prime}},\left(\alpha^{\prime}>\alpha\right)$. Thus, in all cases $\lim _{x \rightarrow \infty} T / T^{\prime}=0$. This contradicts the assumption that $C e\left[x l_{n}(x)\right]$ is a solution of (1).

THEOREM 3. No solution of an algebraic difference equation of the mth order can exceed a function $\mathrm{Ce}_{2}\left[x l_{n}(x)\right]$ for all $x>x_{0}(n)$, if the terms of the equation are so related that when $\beta_{m}^{\prime}=\beta_{m}$ then $\beta_{i}^{\prime} \geqq \beta_{i}$, $(i=0,1,2, \cdots, m-1)$.

${ }^{6}$ It is interesting to observe that from this fact we can obtain a proof that a solution of a first order algebraic differential equation cannot equal or exceed $e_{2}\left[x l_{n}(x)\right]$ for all $x \geqq x_{0}(n)$. For, when the interval of difference, say $\omega$, is reduced, the intervals for which $y(x)$ may exceed $e_{2}\left[x l_{n}(x)\right]$ are also reduced. In the limit as $\omega \rightarrow 0$, the length of these intervals approaches zero and the difference equation approaches a differential equation. Hence a solution of an algebraic differential equation could be at most tangent to $e_{2}\left[x l_{n}(x)\right], x \geqq x_{0}(n)$. However, Hardy, Lindelöf and Borel have established lower bounds for the rate of increase of the solutions of an algebraic differential equation. 
Proof. Assume that $y(x)$, a solution of (1), is greater than or equal to $C e_{2}\left[x l_{n}(x)\right]$ for $x>x_{0}(n)$. Under the hypothesis of the theorem the sequences (4) are of two general types: either the first term is positive and the other terms are arbitrary, or the first term is zero and all the other terms, except the last, are greater than or equal to zero. Corresponding to these two types of sequences the ratios $T / T^{\prime}$ take the forms

$$
B\left\{\frac{x^{K_{0}} y(x)^{K_{1}} y(x+1)^{K_{2}} \cdots y(x+m-1)^{K_{m}}}{y(x+m)}\right\}^{\beta_{m^{\prime}-\beta_{m}}}
$$

and

$$
B\left\{\frac{x^{k_{0}}}{y(x)^{k_{1}} y(x+1)^{k_{2}} \cdots y(x+i)^{k_{i-1}}}\right\}
$$

respectively, where $K_{0}, K_{1}, K_{2}, \cdots, K_{m}$ are rational numbers, $k_{1}, k_{2}, \cdots, k_{i-1}$ are positive integers or zero, and $k_{0}$ is any positive or negative integer unless all $k_{j}=0, j=1,2, \cdots, i-1$. The second set of ratios all approach zero as $x$ increases indefinitely. Hence, not all of the ratios of the first type do approach zero or otherwise $y(x)$ is not a solution of (1). Of the finite number of terms of this type there is a maximum value for $K_{0}$, a maximum value for $K_{1}, \cdots$. Now consider the ratio

$$
\left\{\frac{x^{M_{0}} y(x)^{M_{1}} y(x+1)^{M_{2}} \cdots y(x+m-1)^{M_{m}}}{y(x+m)}\right\},
$$

where $M_{i}$ is the maximum value of all the $K_{i}$ for the ratios of type (9). Some positive constant power of this expression is greater, for $x>x_{0}(n)$, than the absolute value of any of the ratios $T / T^{\prime}$. And since some of the ratios do not approach zero,

$$
\frac{x^{M_{0}} y(x)^{M_{1}} y(x+1)^{M_{2}} \cdots y(x+m-1)^{M_{m}}}{y(x+m)}>\frac{1}{N} .
$$

That is,

$$
y(x+m) \leqq N x^{M_{0}} y(x)^{M_{1}} \ldots y(x+m-1)^{M_{m}}, \quad x \geqq x_{0}(n),
$$

or

$$
y(x+m) \leqq N[x y(x) \cdots y(x+m-i)]^{M}, \text { where } \quad M \geqq 2, M_{j},
$$

It follows from this that

$$
(j=0,1, \cdots, m) \text {. }
$$


$y\left(x_{0}+p+m\right) \leqq\left(x_{0}+p\right)^{(M+1)^{p+2}}\left[N y\left(x_{0}\right) \cdots y\left(x_{0}+m-1\right)\right]^{(M+1)^{p+1}}$,

Thus,

$$
p=1,2,3, \cdots \text {. }
$$

$$
\begin{aligned}
& \lim _{p \rightarrow \infty} \frac{y\left(x_{0}+p+m\right)}{C e_{2}\left[\left(x_{0}+p+m\right) l_{n}\left(x_{0}+p+m\right)\right]} \\
& \quad \leqq \lim _{p \rightarrow \infty} \frac{\left(x_{0}+p\right)^{(M+1)^{p+2}}\left[N y\left(x_{0}\right) \cdots y\left(x_{0}+m-1\right)\right]^{(M+1)^{p+1}}}{C e_{2}\left[\left(x_{0}+p+m\right) l_{n}\left(x_{0}+m+p\right)\right]}=0 .
\end{aligned}
$$

But this is contrary to the hypothesis that $y(x)$ is greater than $\mathrm{Ce}_{2}\left[x l_{n}(x)\right]$ for $x>x_{0}(n)$. Therefore if the terms of equation (1) are so related that $\beta_{i}^{\prime} \geqq \beta_{i},(i=0,1,2, \cdots, m-1)$, for every term $T$ for which $\beta_{m}=\beta_{m}^{\prime}$, then a solution cannot remain greater than $C e_{2}\left[x l_{n}(x)\right]$ for all $x>x_{0}(n)$.

In Theorem 3, the difference equation was restricted but there were no restrictions as to the regularity of increase of the solution. Now we consider the general difference equation but make restrictions on the solutions.

THEOREM 4. If $y(x)$ is a solution of an algebraic difference equation of the mth order and if there exists a constant such that the ratio $y(x)^{K} / y(x+1)$, where $K$ is any rational number, gives a monotone sequence as $x$ ranges over the values $a, a+1, a+2, \cdots$, then $y(x)$ cannot exceed $\mathrm{Ce}_{2}\left[x l_{n}(x)\right]$, for any integer $n$, for all $x>x_{0}(n)$.

The proof follows immediately from the above considerations. The ratios $T / T^{\prime}$, corresponding to the $(m+1-i)$ th case for the sequence (4), are of the form

$$
B\left\{\frac{x^{K_{0}} y(x)^{K_{1}} \cdots y(x+i-1)^{K_{i}}}{y(x+i)}\right\}^{\beta_{i^{\prime}-\beta_{i}}} .
$$

When the conditions of the theorem are satisfied and $y(x)$ is greater than $C e_{2}\left[x l_{n}(x)\right]$ for all $x$, then each of the ratios

$$
\frac{[y(x+i-1)]^{K}}{y(x+i)}, \quad i=1,2, \cdots, m,
$$

gives a monotone decreasing sequence as $x$ takes on the values $x_{1}=a$ $+m-1, x_{2}=a+m, x_{3}=a+m+1, \cdots$ Moreover, $\lim _{x \rightarrow \infty} x^{K_{0}} / y(x)=0$. Hence a ratio $T / T^{\prime}$ consists of a product of $x^{K_{0}} / y(x)$ times ratios which decrease monotonically as $x$ ranges over the values $x_{2}, x_{3}, x_{4}, \cdots$. Thus, $\lim _{x_{i} \rightarrow \infty} T / T^{\prime}=0$, for all possible ratios, and this contradicts the assumption that $y(x)$ is a solution of (1). 
DEFINITION. A function $f(x)$ shall be said to be a regularly increasing function, if for every rational value of $K,[f(x)]^{K} / f(x+1)$ is a monotonic function $x>x_{0}$.

COROLLARY. If a solution $y(x)$ of an algebraic difference equation is a regularly increasing function, then

$$
|y(x)|<e_{2}\left[x l_{n}(x)\right], \quad x>x_{0}(n) .
$$

The proof is evident.

We have shown that a regularly increasing solution of an algebraic difference equation cannot increase as rapidly as the function $e_{2}\left[x l_{n}(x)\right]$. The question naturally arises: Is there a function with a slower rate of increase which is also a bounding function for the regularly increasing solutions of algebraic difference equations? Since there are solutions of difference equations that increase as rapidly as the function $a^{b^{x}}$, where $a$ and $b$ are arbitrary numbers, ${ }^{7}$ it is clear that a bounding function for all regularly increasing solutions of all algebraic difference equations must increase more rapidly than a function of the form $e_{1}\left(c x^{m} e^{K x}\right)$. Therefore, a bounding function must increase as rapidly as $e_{2}(v)$, where $v=x p(x)$ and $\lim _{x \rightarrow \infty} p(x)=\infty$. Hence, if there existed a better bounding function than $e_{2}\left[x l_{n}(x)\right]$ it would be of the form $e_{2}(v)$, where $p(x)$ increases slower than any $l_{n}(x)$. Hardy in Orders of Infinity makes the following statement: "It is possible to define functions whose increase . . . is slower than that of any $l_{n}(x)$; but this is not possible if we confine ourselves to functions defined by a finite and explicit formula involving the ordinary functional symbols of analysis." Therefore, if we confine ourselves to the ordinary functional symbols, we have obtained the best bounding function.

Now let us compare our results for the rate of increase of solutions of difference equations with those known for differential equations. The corollary above corresponds in some respects to the unpublished results of Vijayaraghavan relative to the maximum rate of increase of a solution of an algebraic differential equation. He states at the close of a note in the Comptes Rendus des Séances de l'Académie des Sciences: "If $y(x)$, a function defined for all positive values of $x$, is a solution of an algebraic differential equation of the $m$ th order, and if the increase of $y$ satisfies certain very restrictive regularity conditions, then for $x>x_{0},|y(x)|<e_{m}\left(k x^{\Delta}\right)$, where $e_{m}(x)$ designates the $m$ th iterate of the exponential function and $k$ and $\Delta$ are properly determined constants." If the regularity conditions of the two results

${ }^{7}$ A solution of $y(x+1)=y(x)^{b}, b$ a rational number, is $y(x)=a^{b^{x}}$, where $y(0)=a$. 
are comparable, we see that the upper bound for the regularly increasing solutions of a differential equation for $m>1$ is much greater than that for the regularly increasing solutions of a difference equation. It is interesting to observe that there actually are differential equations of higher than first order which have regularly increasing solutions that increase more rapidly than any regularly increasing solution of any algebraic difference equation. In order to see this it is sufficient to verify that the differential equation

$$
x y y^{\prime \prime}-x\left(y^{\prime}\right)^{2}-\left(2 x^{2}+1\right) y y^{\prime}=0
$$

is satisfied by $y(x)=e_{2}\left(x^{2}\right)$.

The story is quite different for equations of first order. There are regularly increasing solutions of some first order algebraic difference equations that increase more rapidly than any solution of any first order differential equation. For, ${ }^{3}$ the solutions of a first order differential equation cannot increase as rapidly as $e_{2}(x)$ and, as was mentioned before, there are solutions of some difference equations of the first order that increase as rapidly as $a^{b^{x}}$, where $a$ and $b$ are arbitrary constants.

The above regularity conditions are very stringent. Are such regularity conditions necessary? As a partial answer to this question, we now show that unless some regularity conditions are imposed on the solutions, there is no bounding function for the solutions of all algebraic difference equations of higher than first order. The following theorem and its proof are modelled after the analogous theorem for differential equations that was given by Vijayaraghavan. ${ }^{3}$

THEOREM 5. For a given function $\Phi(x)$ with an arbitrary rate of increase, there exists a second order algebraic difference equation

$$
P(y(x), y(x+1), y(x+2), x)=0
$$

that is satisfied for all real values of $x$ by a real and continuous function $f(x)$, where $|f(x)|>\Phi(x)$ for an infinite number of values $x_{i},\left\{x_{i}\right\} \rightarrow \infty$.

Proof. We form the function

$$
f(z)=(1 / 2)[\wp(\alpha z)+\overline{\wp(\alpha \bar{z})}],
$$

where $\wp(z)$ is the Weierstrass $\&$-function with the two fundamental periods $\omega$ and $\omega^{\prime}, \bar{z}$ is the conjugate of $z$, and $\alpha=\omega+i \eta$, where $\eta$ is yet to be determined.

We choose $\eta$ real in such a manner that for an infinite number of integral values $n_{j}$ and $p_{j}$ 


$$
\left|p_{j}-n_{j} \eta / \omega^{\prime}\right|<\frac{1}{2 \omega^{\prime}\left[\Phi\left(n_{j}\right)\right]^{1 / 2}} .
$$

For this it is sufficient to take for $\eta / \omega^{\prime}$ a continued fraction in which the denominators of the partial quotients increase in a sufficiently rapid manner.

For real values of $z, f(z)$ is the real part of $\wp(\alpha z)$; hence it is a real function. If $z=n_{j} \omega+p_{j} \omega^{\prime} i+t i$, where $t$ is real and very small, the real part of $8(z)$ is asymptotic to $-1 / t^{2}$. Therefore, it follows from the inequality (11) that for $z=n_{j}$ and for $j$ sufficiently large

$$
-f\left(n_{j}\right) \sim \frac{1}{\left(n_{j} \eta-p_{j} \omega^{\prime}\right)^{2}}>\Phi\left(n_{j}\right) .
$$

Now by virtue of the addition formula for $\wp(z)$,

$$
\overline{\wp(\alpha \bar{z})}=2 f(z)-\wp(\alpha z)
$$

satisfies a first order algebraic difference equations. If $\wp(\alpha z)$ is eliminated between the difference equation for $2 f(z)-\wp(\alpha z)$ and the one obtained from it by replacing $z$ by $z+1$, we obtain a second order algebraic difference equation that is satisfied by the real function of $f(z)$ for all real values of $z$. This completes the proof.

UNIVERSITY OF MARYLAND 